\title{
Uncoupling protein-2 (UCP2): une nouvelle protéine mitochondriale contrôlant la production d'ATP et de chaleur, et un nouveau gène impliqué dans l'obésité
}

L'oxydation mitochondriale des substrats (en fait la réoxydation des coenzymes réduits) est couplée à la phosphorylation de l'ADP. Ce processus de phosphorylation oxydative, contrôle la synthèse d'ATP et l'équilibre énergétique de toutes les cellules animales. En fait, le niveau de couplage de la phosphorylation oxydative est plutôt faible, puisque seulement $50 \%$ de l'énergie libre venant des oxydations mitochondriales est utilisée pour phosphoryler l'ADP. Une partie de l'énergie des oxydations permet le transport d'anions dans la matrice mitochondriale et l'échange ADP/ATP, une autre partie étant dissipée sous forme de chaleur par des mécanismes non expliqués.

Certaines cellules particulières dissipent la quasi-totalité de l'énergie respiratoire sous forme de chaleur lorsqu'elles sont activées: il s'agit des adipocytes bruns, cellules thermogéniques actives chez les mammifères à la naissance, lors de l'exposition au froid, ou lors du réveil chez les hibernants. Dans ce cas, la chaleur est due à UCP (uncoupling protein), une protéine mitochondriale spécifique des adipocytes bruns, qui active et découple leur respiration [1]. Sachant que toute mitochondrie oxydant des substrats dans n'importe quel type cellulaire produit de la chaleur par un mécanisme inconnu, on pouvait faire l'hypothèse que ce mécanisme impliquait une protéine semblable à l'UCP mitochondriale des adipocytes bruns. Cette hypothèse a conduit Daniel Ricquier et Serge Raimbault (Cnrs à Meudon) à cribler une banque d'ADNc de muscle squelettique de souris avec l'ADNc $U C P$, et à cloner un $\mathrm{ADNc}$ codant pour une protéine homologue de l'UCP [2]. Cette nouvelle protéine a été appelée UCP2, l'UCP des adipocytes bruns devenant UCP1. La protéine UCP2 est pour $59 \%$ de sa séquence identique à UCP1. L'expression d'UCP2 dans des levures démontra que la protéine UCP2 affecte le potentiel membranaire mitochondrial et décroît le niveau de couplage de la respiration (travail de Christophe Fleury, Corinne Lévi-Meyrueis et Frédéric Bouillaud). Ainsi UCP2 pourrait expliquer, ou contribuer à expliquer, pourquoi le niveau de couplage des oxydations mitochondriales à la phosphorylation de l'ADP, est limité.

Parallèlement à ce travail et de manière indépendante, Craig Warden clona aussi l'ADNc de UCP2 au même moment, à l'Université de Davis (CA, USA). Les groupes de Meudon et de Davis analysèrent l'expression tissulaire du messager $U C P 2$ chez les rongeurs et l'homme, et constatèrent sa présence dans la plupart des organes et de nombreuses lignées cellulaires. Cet ARN messager est présent, en particulier, dans les tissus adipeux blanc et brun, les muscles, le cœur, les reins, le placenta. Les organes et cellules les plus riches en messager UCP2 sont la rate, la moelle osseuse, le thymus, les poumons et l'estomac (organes riches en macrophages), les lymphocytes et les macrophages. Une telle distribution pourrait expliquer certains mécanismes particuliers aux macrophages et suggère un rôle d'UCP2 dans les processus inflammatoires et la fièvre.

Ayant précédemment repéré des locus chromosomiques impliqués dans l'obésité spontanée ou induite par l'alimentation chez la souris, Craig Warden observa que la localisation du gène UCP2 [2] coïncidait avec des QTL (quantitative trait locus) pour l'obésité dans au moins trois modèles indépendants de souris, et correspondait à une même région du chromosome 7 , région liée génétiquement à l'insulinémie et à la glycémie. Chez l'homme, le gène UCP2 est localisé en 11q13.

Par ailleurs, Richard Surwit, à l'Université de Duke (Durham, NC, USA), avait démontré que les souris $\mathrm{A} / \mathrm{J}$ étaient résistantes à l'obésité induite par un régime hyperlipidique, ces souris ayant une «efficacité alimentaire» (gain de poids/calories ingérées) réduite. En dosant le messager UCP2, Surwit et Collins, observèrent des niveaux de ce messager plus élevés dans le tissu adipeux blanc de ces souris que chez des souris B6 qui, elles, développent une obésité lors d'un régime hyperlipidique. En outre, les souris $\mathrm{A} / \mathrm{J}$ soumise à un tel régime surexpriment le messager $U C P 2$ nettement plus que les souris B6 [2].

Ainsi, UCP2, protéine dissipatrice d'énergie, pourrait expliquer la résistance (ou la tendance) à l'obésité, en contrôlant la thermogenèse induite par l'alimentation, et l'efficacité métabolique. Il s'agit là d'un nouveau gène et d'une nouvelle protéine d'intérêt pour la recherche sur les obésités, le diabète non insulino-dépendant, les cachexies, les processus thermogéniques associés aux processus inflammatoires, ainsi que les troubles de la thermorégulation. Enfin, UCP2 pourrait expliquer en partie le mécanisme effecteur de la fièvre.

D.R.

F.B.

1. Ricquier $D$. Thermogenèse et obésité: mécanismes moléculaires. Med Sci 1985; 1 : 147-53. 2. Fleury C, Neverova M, Collins S, Raimbault S, Champigny O, Levi-Meyrueis C, Bouillaud F, Seldin MF, Surwit RS, Ricquier D, Warden CH. Uncoupling Protein-2 : a new thermogenic protein and a new gene linked to obesity and hyperinsulism. Nature Genet 1997; 15 : 269-72. 